\title{
A Tracer Gas Leak Rate Measurement Method for Circular Air Circuits
}

\author{
Arne Tiddens ${ }^{\mathrm{a}, *}$, Marc Röger $^{\mathrm{b}}$, Hannes Stadler $^{\mathrm{a}}$, Bernhard Hoffschmidt $^{\mathrm{c}}$ \\ ${ }^{a}$ Institute of Solar Research, German Aerospace Center (DLR), Karl-Heinz-Beckurts-Str.13, 52428 Jülich, Germany \\ ${ }^{b}$ Institute of Solar Research, German Aerospace Center (DLR), Plataforma Solar de Almería, Tabernas 04200, Spain \\ ${ }^{c}$ Institute of Solar Research, German Aerospace Center (DLR), Linder Hoehe, 51147 Cologne, Germany
}

\begin{abstract}
Qualitative measurements of leaks in air flows are frequently conducted in engineering. However, an environmentally friendly leak rate measurement technique which yields quantitative results and is applicable to hot, large-scale circular air flows $\left(\approx 10 \mathrm{~kg} / \mathrm{s} ; \approx 700^{\circ} \mathrm{C}\right)$ with high leak rates has not been presented yet. This paper describes the development, test and validation of a stationary and a dynamic, helium based tracer gas method on a lab-scale model of a solar air receiver with partial air recirculation. Helium is chosen as tracer gas since it is environmentally friendly, stable under high temperatures and is also cheap for large-scale air flows. The tracer gas is injected either continuously or intermittently into the model system, its concentration is measured using a mass spectrometer and the static or dynamic concentration response of the system is used to determine the leak rate. The stationary method needs two measurement points upstream and downstream the leak, the dynamic method only one measurement point if applied to a circular air flow system. Since the dynamic method is time dependent the transfer function of the measurement setup was determined and the dynamic measurement error considered. An extensive uncertainty analysis is presented for both the stationary and dynamic method. Exemplary measurements were conducted at the model system with very good results. Both dynamic and static measurements yield the same result within their confidence intervals. The leak rate of the solar receiver model with a mass flow rate of $\dot{m}_{i n}=(0.247 \pm 0.008) \mathrm{kg} / \mathrm{s}$ was measured to be $l_{\text {stat }}=(36.1 \pm 2.3) \%$ and $l_{\mathrm{dyn}}=(34.5 \pm 3.6) \%$ with the static and dynamic method respectively.
\end{abstract}

Keywords: Air return ratio, Tracer gas, Circular tracer, Leak rate, Solar air receiver, Air receiver, Measurement technique, Dynamic mass spectroscopy

\section{Introduction}

Concentrated solar energy provides an environmentally friendly and virtually unlimited source of high-temperature heat [12]. As in most renewable energy research, cost reduction is the main research goal. It is therefore essential to quantify all factors that influence the efficiency of energy production. The examined open volumetric receiver concept was build on a large scale as the Solar Tower Jülich demonstration solar power plant [10]. Here the sunlight is reflected and concentrated by a field of heliostats onto an open volumetric air receiver. This open volumetric receiver consists of a porous ceramic structure which is heated up by absorbing the sunlight creating surface temperatures of up to $1000^{\circ} \mathrm{C}$. Air is sucked through the absorber modules to transfer the thermal energy to the steam boiler, where a conventional power block is used to produce electricity. Due to the low heat capacity of air, high air mass flows are needed. After the air passes the steam boiler, it has a temperature of up to $200^{\circ} \mathrm{C}$ and is returned to reuse the residual heat. It is blown out through the

\footnotetext{
${ }^{*}$ Corresponding author

Email address: Arne.Tiddens@dlr.de (Arne Tiddens)
}

structure of the receiver and is partially sucked in again. The fraction of the blown out air which is sucked in again, is called air return ratio (ARR) (Eqn. 1). At this point the examined leak of the system occurs.

To achieve a high receiver efficiency it is important to decrease this leak in the air circuit. It depends on many variables such as wind, geometry of the receiver design and operational mode and is so far unknown. Since this leak can be reduced by a multitude of measures, it is of vital importance to be able to measure it [16, 15].

The leak rate measurement can be approached either by measuring the mass flow field in front of the receiver, or measuring the temperature or a chemical property of the air before and after the leak which changes according to the size of the leak. To measure the air flow field in front of the receiver the measurement technique need to be applicable with very high precision. However, most flow measurement techniques are not employable on the large scale of solar tower power plants or do not yield quantitative results. An existing and possibly the most feasible flow measurement is the laser based Particle Image Velocimetry (PIV). It would be possible on such a scale, however resulting in large uncertainties.

Due to the difference in temperatures before and after 
the receiver, caused by the leak of the system, an energy balance seems possible measuring only the air flows and their temperature. On the outside of the receiver this measurement is not feasible, since for a measurement of the air flows and their temperature thousands of thermocouples and mass flow measurements would be needed. A measurement in the airflow before and after the receiver as conducted by Tellez et al. [14 does not lead to correct results, since the outlet temperature is not well defined. This occurs because the outlet air is heated by the outsides of the absorber cups while it is blown out [1].

Due to the above mentioned difficulties, we decided to use a tracer gas method, whereby a tracer gas is injected and measured in the air flow. This is the only possibility, since all other measurable, intrinsic properties of the air are correlated to the temperature.

Tracer gas measurements are widely used in medicine, in ventilation experiments for buildings and air-conditioning systems. Inert tracer gas washout tests are for example used to perform extended lung function tests [11. In contamination experiments Tang et al. [13] use a tracer to simulate the spreading of diseases in hospitals. Similarly the spreading of hot or cold air is quantified in buildings [7. For example Ghazi and Marshall [6] use a carbon dioxide tracer gas to determine and characterize leaks across windows, Cui et al. 22 use a decay rate method to determine the air change rate of buildings. These measurements however are not transferable to the described measurement environment, due to the harsh conditions at the Solar Tower Jülich. The high circular air mass flows with large leak rates do not allow for any gases already present in air or environmentally harmful gases. The occurring surface temperatures further limit the possible tracer gas candidates. The commonly used $S F_{6}$ can for example only be heated without decomposition up to $500{ }^{\circ} \mathrm{C}$ in the absence of catalytic metals and has furthermore the highest global warming potential of all gases [9, 5].

For development and validation purposes, we constructed a 1:2 scale model of a sub-receiver ( 56 absorber modules) and the corresponding air circuit of the Solar Tower Jülich (1080 absorber modules). The circular nature of the measurement environment permits additionally to the static measurement a dynamic measurement. The latter allows a quantitative leak rate measurement with just one dynamic tracer gas concentration measurement. By comparison of the static and dynamic methods, a validation of the methods of measurement at a laboratory scale can be achieved. This paper describes the development, application and validation of the two tracer gas methods.

\section{Experimental Set-up}

A model of a part of the open volumetric receiver structure has been built to develop, test and validate the measurement setup without solar irradiation. It is a model of the open volumetric receiver containing 9x6 absorber cups at a scale of 1:2. Its schematic is shown in Fig. 2, a photo

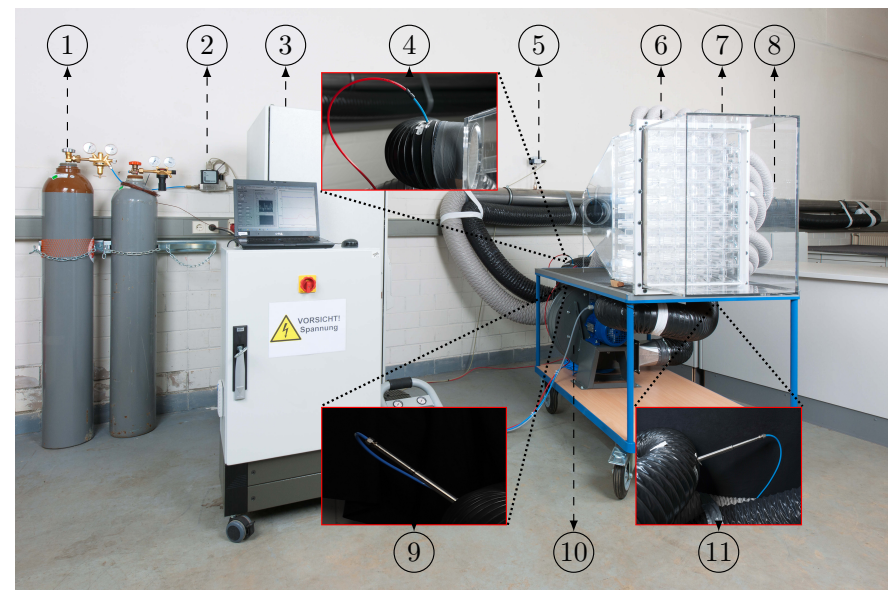

Figure 1: Photo of the measurement setup.

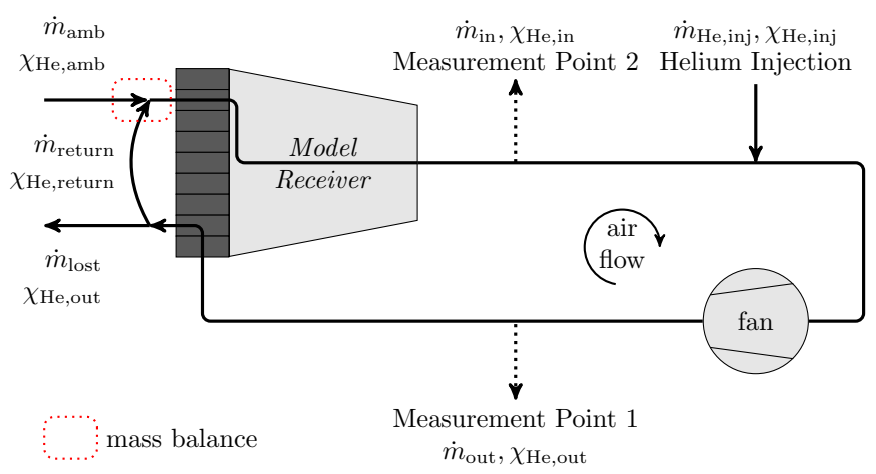

Figure 2: Schematic diagram of the model

in Fig. 1. The air is sucked through the receiver (6) by a fan (10) and is then returned to the receiver front through 13 air return tubes (8). The model is designed regarding the theory of similarity, to produce a flow pattern in front of the receiver similar to that of the Solar Tower Jülich. Due to the smaller size, modifications to the air circuit are simpler than at the full scale solar power plant. The fan can be operated at different frequencies to control the air mass flow. The air mass flow is measured to allow the measurement with different air mass flow rates by a thermal flow mass sensor (5). The receiver can be covered by a removable lid (7), to test for unwanted leaks. By closing the receiver and removing some of the 13 return air tubes, different scenarios with fixed, unknown leak rates can be created for validation purposes (sec. 4.3).

To conduct a tracer gas measurement, helium (1) is injected into the system at (4). The helium mass flow is controlled using a mass flow controller (2). The resulting helium concentration is then measured by extracting a sample at either point of measurement (9), (11). Due to the choice of helium as tracer gas (see sec. 3.1) and the low concentrations that need to be measured, a quadrupole mass spectrometer (QMS 200, Pfeiffer Vacuum) (3) is used to determine the concentration. 


\begin{tabular}{|c|c|c|c|c|c|c|}
\cline { 2 - 7 } \multicolumn{1}{c|}{} & $\mathrm{Ar}$ & $\mathrm{CO}_{2}$ & $\mathrm{He}$ & $\mathrm{H}^{*}$ & $\mathrm{SF} 6$ & $\mathrm{Ne}$ \\
\hline natural conc. & -- & -- & + & ++ & ++ & ++ \\
\hline pollution & ++ & $\mathrm{o}$ & ++ & + & -- & ++ \\
\hline therm. stability & ++ & ++ & ++ & -- & -- & ++ \\
\hline price & + & ++ & - & ++ & - & -- \\
\hline
\end{tabular}

Table 1: The table shows different tracer gas candidates and their suitability according to different aspects. $\mathrm{H}^{*}$ stands for forming gas, which is a mixture of $95 \%$ nitrogen and $5 \%$ hydrogen.

\section{Theory}

\subsection{Tracer Gas Method}

The high temperatures, high air mass flow, openness and presence of concentrated solar radiation of the future measurement environment pose requirements on the choice of tracer gas. The tracer gas must hence be environmentally friendly, stable under high temperatures and may only occur in air in low concentrations (see Table 1). This reduces the choice of tracer gases down to the noble gases. Due to economic reasons we chose helium.

The relative leak rate of the system per circulation is defined as

$$
l=1-\frac{\dot{m}_{\text {return }}}{\dot{m}_{\text {out }}}=1-A R R
$$

whereby $\dot{m}_{\text {out }}$ is the air mass flow leaving before the leak, and $\dot{m}_{\text {return }}$ is the part of this air which is returned to the air circuit. $A R R$ is the previously mentioned air return ratio, which is defined by Ahlbrink et al. [1] as $A R R=$ $\dot{m}_{\text {return }} / \dot{m}_{\text {out }}$.

Under the assumption that the amount of injected helium is small compared to the airflow, it can be assumed that the molar mass of the examined air flows are equal. Furthermore, it can be approximated that $\dot{m}_{\text {out }}=\dot{m}_{\text {in }}$. A mass balance as indicated in Fig. 2 results in

$$
\dot{m}_{\text {return }}+\dot{m}_{\mathrm{amb}}=\dot{m}_{\mathrm{in}}=\dot{m}_{\mathrm{out}} .
$$

The helium mass balance at the indicated point is

$$
\dot{m}_{\mathrm{return}} \cdot \chi_{\mathrm{He}, \mathrm{return}}+\dot{m}_{\mathrm{amb}} \cdot \chi_{\mathrm{He}, \mathrm{amb}}=\dot{m}_{\mathrm{in}} \cdot \chi_{\mathrm{He}, \mathrm{in}},
$$

whereby $\chi_{\mathrm{He}}$ is the helium concentration at different locations in the air circuit as shown in Fig. 2,

To be able to determine the leak rate $l$ from helium concentrations, the helium concentration of the return air which is blown out $\chi_{\mathrm{He} \text {,out }}$, must be the same as the helium concentration of the return air being sucked in again $\chi_{\mathrm{He} \text {,return }}$. In front of the receiver the blown out air is mixed by dispersion as well as diffusion. Since the mixing is turbulent that occurs, the dispersion is much faster than the diffusion. This arises from the different origin of dispersion and diffusion in turbulent flow. Whereas diffusion is caused by the small-scale Brownian motion, the turbulent dispersion is caused by gusts and eddies [3. Since dispersion is therefore the dominant cause of mixing, and

\begin{tabular}{|c|c|c|c|c|}
\hline$\dot{\mathrm{m}}_{\text {in }}[\mathrm{kg} / \mathrm{s}]$ & \multicolumn{4}{|c|}{ Leak rate [\%] } \\
\hline \hline & 1 & 10 & 50 & 100 \\
\hline 0.1 & 0.0001 & 0.0011 & 0.0054 & 0.0109 \\
\hline 1 & 0.001 & 0.011 & 0.054 & 0.109 \\
\hline 10 & 0.01 & 0.11 & 0.54 & 1.09 \\
\hline 100 & 0.1 & 1.1 & 5.4 & 10.9 \\
\hline
\end{tabular}

Table 2: The table shows the number bottles (501, 300bar) needed to achieve a helium concentration of $100 \cdot \chi_{H e, a m b}$ for one hour for different air mass flows $\left(\dot{\mathrm{m}}_{i n}\right)$ and leak rates.

furthermore has no effect on $\chi_{\mathrm{He} \text {,return, the effect of diffu- }}$ sion can be neglected, causing $\chi_{\mathrm{He}, \text { return }}=\chi_{\mathrm{He} \text {,out }}$.

With Eqs. 23 this leads to

$$
\frac{\dot{m}_{\text {return }}}{\dot{m}_{\text {out }}} \cdot \chi_{\mathrm{He}, \text { out }}+\left(1-\frac{\dot{m}_{\text {return }}}{\dot{m}_{\text {out }}}\right) \cdot \chi_{\mathrm{He}, \text { amb }}=\chi_{\mathrm{He}, \text { in }} .
$$

Combining this with Eqn. 1. the leak rate can be expressed as

$$
l=1-\frac{\chi_{\mathrm{He}, \mathrm{in}}-\chi_{\mathrm{He}, \mathrm{amb}}}{\chi_{\mathrm{He}, \mathrm{out}}-\chi_{\mathrm{He}, \mathrm{amb}}} .
$$

This shows, that the leak rate can be determined measuring only $\chi_{\mathrm{He}, \mathrm{in}}, \chi_{\mathrm{He}, \text { out }}$ and $\chi_{\mathrm{He}, \mathrm{amb}}$, as long as $\chi_{\mathrm{He}, \text { out }} \neq$ $\chi_{\mathrm{He}, \mathrm{amb}}$. To achieve this, helium must be injected into the system.

The locations of the added helium and the concentration measurement are chosen to be applicable at the Solar Tower Jülich and allows the static (sec. 3.2) as well as dynamic (sec. 3.3 measurement techniques to be applied. The location of the point of injection is furthermore chosen, so that the injected helium is mixed by the fan before the next measurement location (see Fig. 2). The helium injection time was minimized to reduce consumption, reduce measurement time and increase temporal resolution without lowering measurement result quality. The amount of helium which is added is small resulting in a low helium concentration of around $\left(1 \%\right.$ o $\left.\approx 200 \cdot \chi_{H e, a m b}\right)$ in the system, which is a concentration that can be realistically achieved at the Solar Tower Jülich. Table 2 shows the amount of helium needed for different scenarios to achieve a helium concentration of $100 \cdot \chi_{H e, a m b}$ for one hour, which is a realistic time frame for several experiments during a day.

\subsection{Static Circular Concentration Measurements}

The simplest way to determine the leak rate via a tracer rate measurement is to inject the tracer gas before the leak and measure the initial concentration at measuring point $1\left(\chi_{\mathrm{He}, \text { out }}\right)$ and the final concentration at measuring point $2\left(\chi_{\mathrm{He}, \text { in }}\right)$ as shown in Fig. 2, After the concentration reaches equilibrium, the point of measurement is switched every two minutes. In Fig. 3 the raw data of an exemplary measurement is shown. The leak rate can then be 


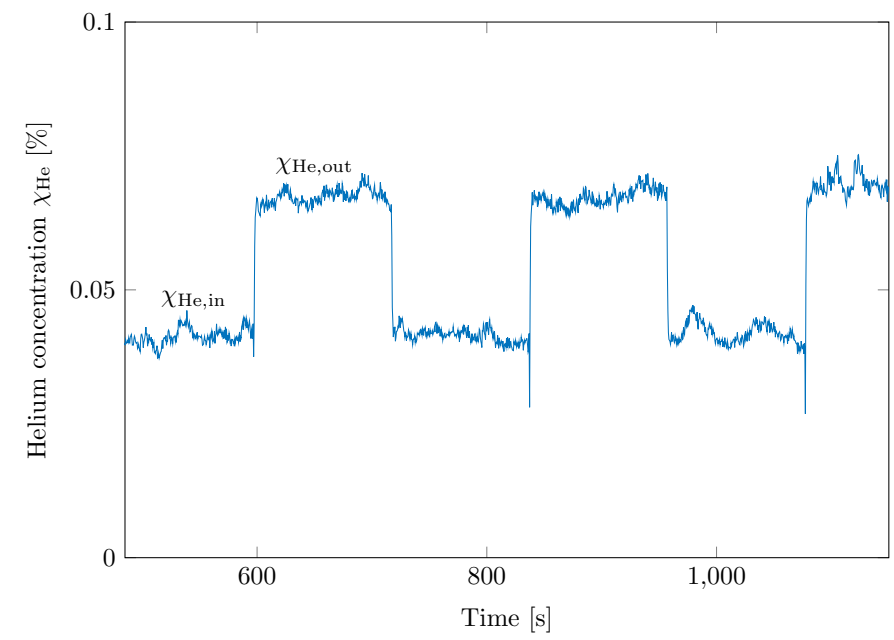

Figure 3: Exemplary helium concentration measurements, switching between the two points of measurement every 120 seconds. Helium is injected continuously during the experiment.

determined directly by using Eqn. 5. However, two further prerequisites have to be examined: The distribution of helium at the measurement points cross sections as well as peripheral leaks outside of the receiver.

\subsubsection{Mixing}

The helium concentration was measured across the cross section of the piping at both points of measurement. At these points the helium concentration is homogeneously distributed and hence single point sampling is possible. The result of an exemplary measurement at measurement point 2 is shown in Fig. 4

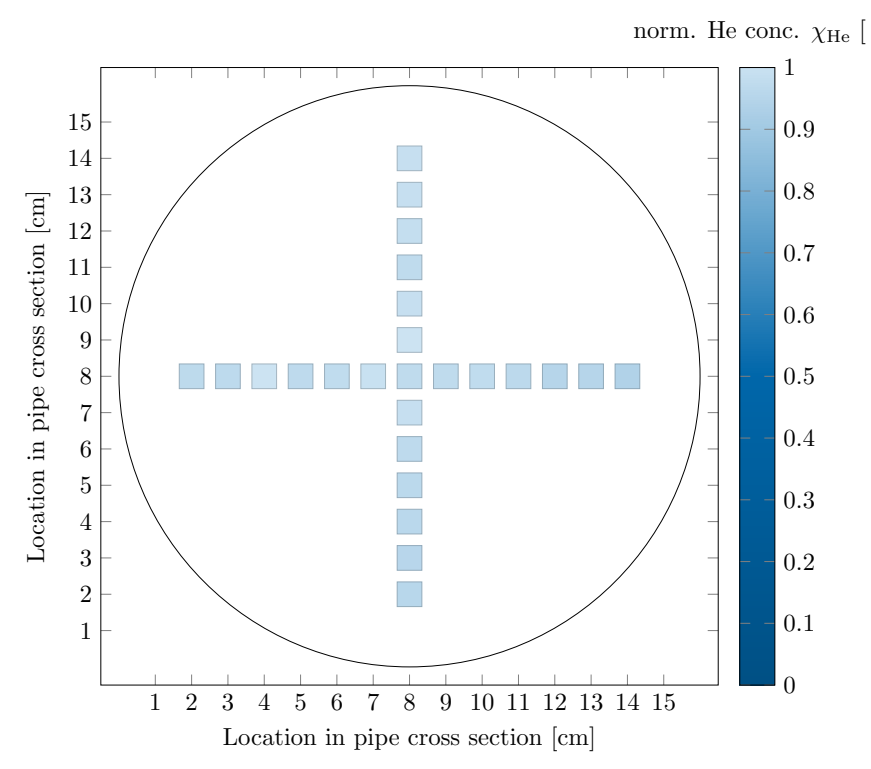

Figure 4: The helium concentration across the piping of the receiver model at measurement point 2 (see Fig. 2 is homogeneously distributed.

\subsubsection{Peripheral Leakage Measurement}

To measure the leak rate of the receiver only, it must be assured that the rest of the air circuit is leak tight. To achieve this, the front of the receiver was sealed (see Fig. 5) and the circuit filled with helium. A qualitative helium leak and sniffing inspection from the outside of the system and counter-measures were conducted to reduce the leakage of the system to a minimum. A leak between the two measurement points could not be observed after optimization of the sealing.

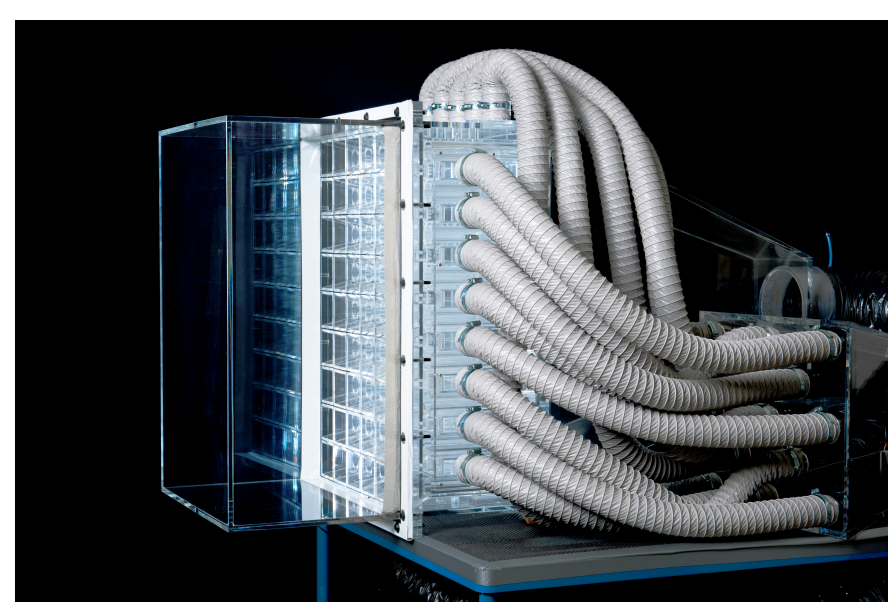

Figure 5: Photo of the receiver model with a closed front with all 13 return tubes attached.

\subsection{Dynamic Circular Concentration Measurements}

The advantage of the dynamic circular concentration measurement is that only one point of measurement is needed. The measurement is conducted by injecting helium with a fixed flow rate and duration into the system and measuring the resulting concentration response over time (see Fig. 6). In contrast to the static measurement, the transient concentration curves are relevant and the complete concentration curve is fitted instead of measuring at equilibrium.

The point of measurement is chosen to be directly behind the blower, since the concentration of helium across the cross section of the piping is homogeneous there. The disadvantage of the dynamic measuring method is that its measurement procedure and the evaluation of the data is more complex than of the static measurement.

In Fig. 7 the theoretical concentration response is shown if dispersion of helium is ignored. The leading edge shows an increase of concentration until the concentration reaches its maximum at equilibrium. As soon as the helium injection is stopped, the helium concentration decreases every cycle with a period length of $T$, by the factor $(1-l)$. The results of a realistic measurement in Fig. 6 show, that the distinguished concentration steps in Fig. 7 disappear and the concentration response of the leading and trailing edge can be described by the exponential growth and 


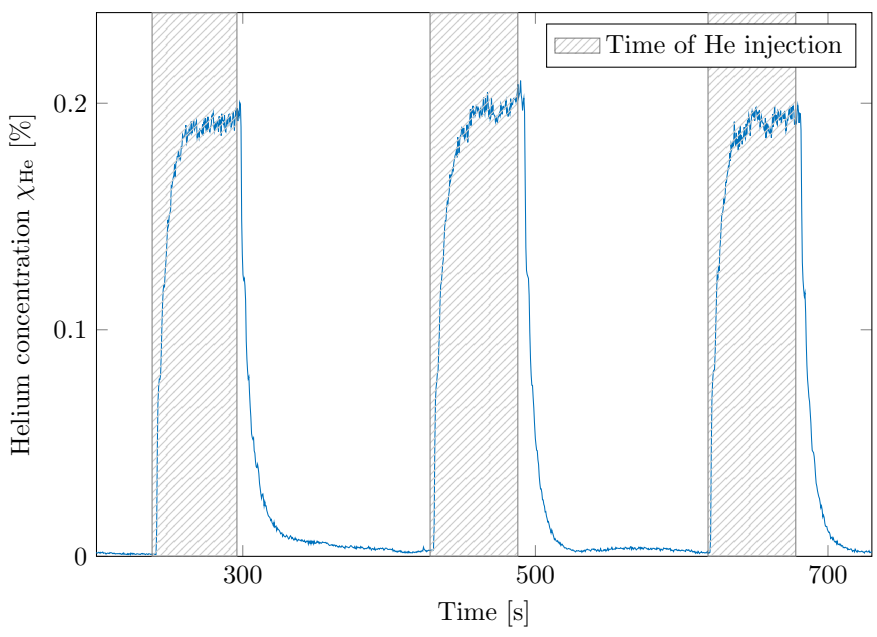

Figure 6: The time of helium injection as well as the helium concentration response due to this injection are shown.

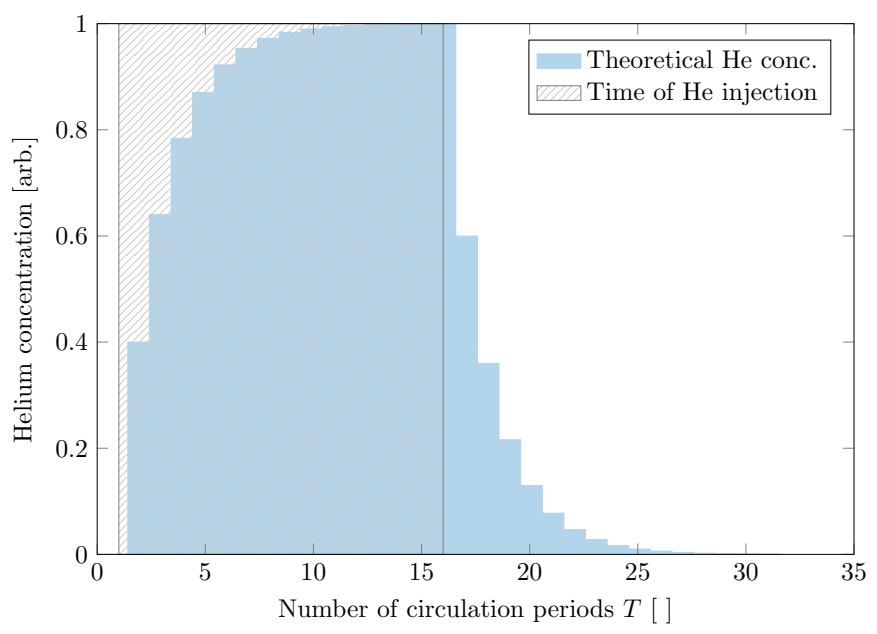

Figure 7: The theoretical helium concentration response of a circular air circuit with a leak rate $l=0.4$, ignoring the dispersion of helium in the system.

decay functions shown in Eqs. 6 and 7 respectively

$$
\begin{aligned}
& \chi_{\text {He,leading }}(t)=A\left(1-\left(1-l_{\text {meas }}\right)^{t / T}\right) \\
& \chi_{\text {He,trailing }}(t)=A\left(1-l_{\text {meas }}\right)^{t / T} .
\end{aligned}
$$

\subsubsection{Measuring the Circulation Period}

The circulation period of the air system $T$ is required to determine $l_{\text {meas }}$ from Eqs. 6 and 7 since it is the only other unknown variable.

The period $T$ can be measured by injecting a concentration peak with the shortest experimental period possible into the system. By measuring the time it takes between two measurements of this concentration peak at the same measurement point, the circulation period is measured. A measurement of the circulation period is shown in Fig. 8 ,

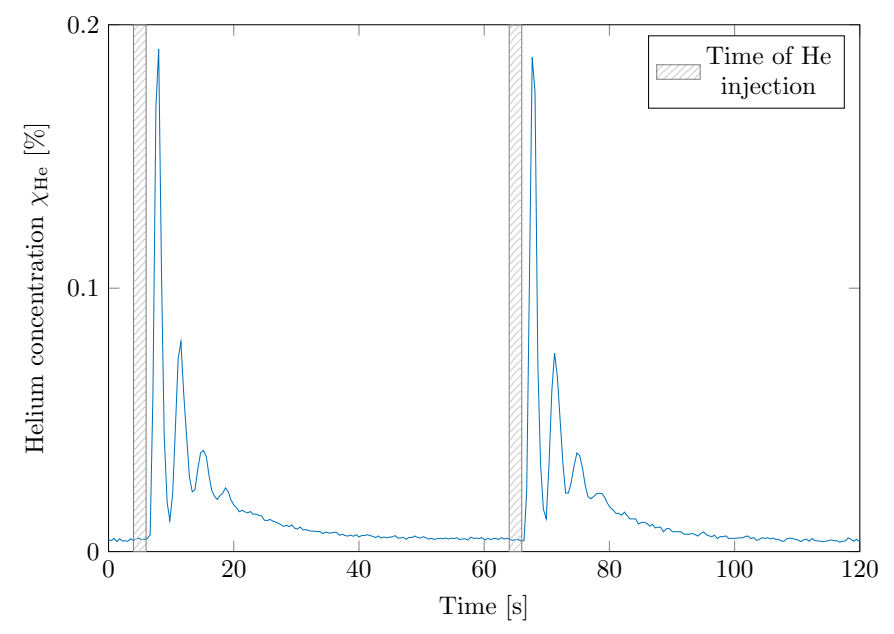

Figure 8: The helium concentration response due to the injection of two short helium peaks are shown. Each peak visibly passes the point of measurement four times, before the peaks become indistinguishable. The time between between two peaks is the circulation period.

\subsubsection{Measurement of the Transfer Function of the Set-up}

The mass spectrometer measurement of the dynamic concentration in sec. 3.3 have to be corrected for its response characteristics. Therefore, the transfer function of the measurement set up must be known to be able to correct the resulting dynamic error. Since dealing with a complicated measurement system, the transfer function is determined by conducting a black box system analysis. By introducing a step function into the system, the transfer function $G(s)$ can be measured since $\mathcal{L}\left(\chi_{\text {step }}(t)\right)=G(s) \cdot s$. Hereby $\mathcal{L}\left(\chi_{\text {step }}(t)\right)$ is the Laplace transformed of the step response $\chi_{\text {step }}(t)$ and the parameter $\mathrm{s}$ is the complex number frequency. The step function is realized experimentally by filling a flask with different helium-air mixtures and covering the flask with a rubber seal. By attaching a plug and needle to the end of the measuring probe, the rubber seal is pierced, the probe enters the flask which is immediately sealed again by the plug (see Fig. 9p. The measured step response is shown in Fig. 10.

As shown in Fig. 10, the transmission element $\mathrm{PT}_{2}$ response is a very good fit for the concentration step response, since the normalized root-mean-square deviation is very close to zero. The transmission element $\mathrm{PT}_{3}$ doesn't yield more accuracy but introduces an unnecessary variable. Therefore the $\mathrm{PT}_{2}$ model is chosen, resulting in a step response in the time domain of

$$
\chi_{\text {He,step,norm }}(t)=1-\frac{T_{1} e^{-\frac{t}{T_{1}}}}{T_{1}-T_{2}}+\frac{T_{2} e^{-\frac{t}{T_{2}}}}{T_{1}-T_{2}},
$$

with $T_{1}, T_{2}$ as fitting parameters. Since we are treating the measurement setup as a black box, the transfer function must be determined at different concentration levels of helium $\chi_{\mathrm{He}, \text { const }}$ in the flask (see Fig. 9). The time delay of the measurement setup was ignored, since it is of no relevance for the measurement. The transfer function in 


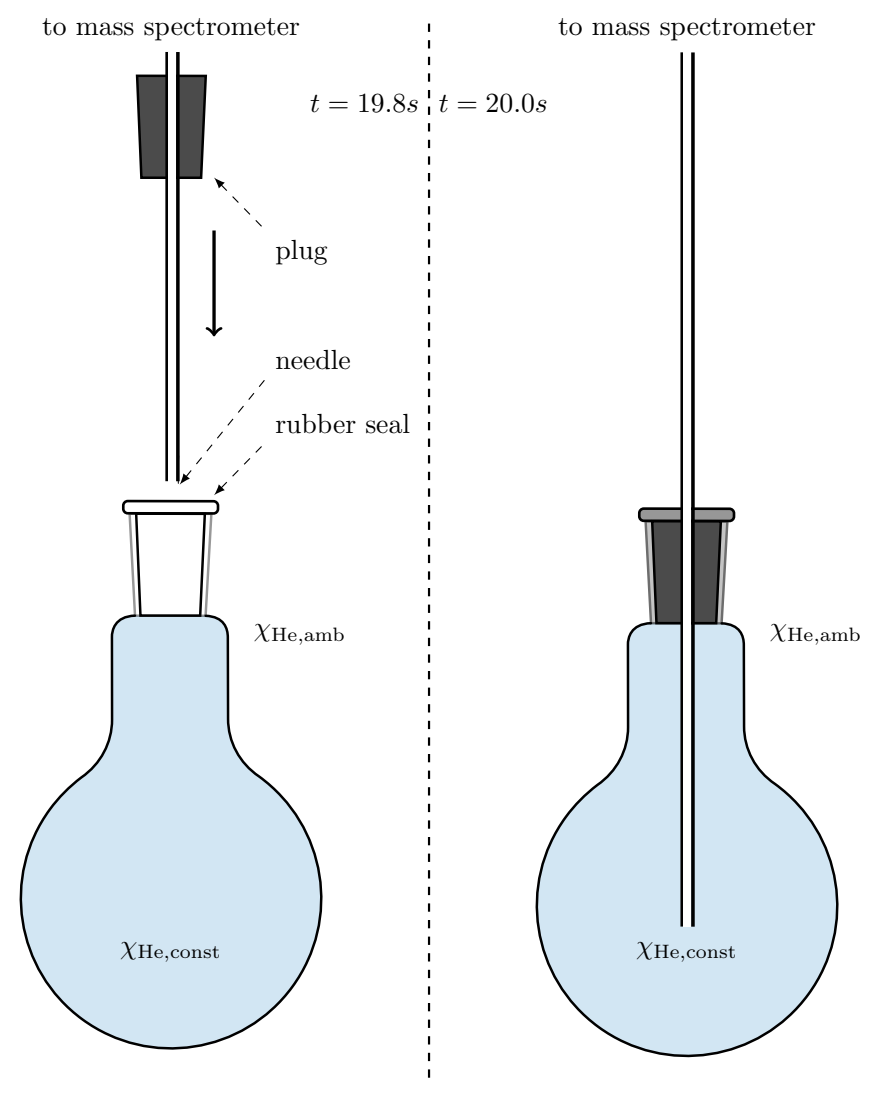

Figure 9: The figure shows the schematic of the creation of a helium concentration step function at the end of the measuring probe. The indicated time corresponds to the time in Fig. 10

frequency domain, corresponding to the $\mathrm{PT}_{2}$ transmission element is found to be

$$
G(s)=\frac{1}{\left(s \cdot T_{1}+1\right)\left(s \cdot T_{2}+1\right)} .
$$

\subsubsection{Compensation of Dynamic Measurement Error}

Since the uncorrected concentration data can be accurately be described by the theoretically expected Eqs. 6 and 7 and the corrected data should also be describable by these equations, the corrected leading edge $\chi_{\text {cor }}$ should be expressible as

$$
\begin{aligned}
\chi_{\text {cor }}(t) & =A\left(1-\left(1-l_{\text {cor }}\right)^{t / T}\right) \\
& =A\left(1-\left(1-l_{\text {meas }}\right)^{t / T} \cdot \operatorname{cor}_{\mathrm{dyn}}\right),
\end{aligned}
$$

with the corrected leak rate $l_{\text {cor }}$. Since the maximum helium concentration has no effect on the relative leak rate. Since this must furthermore be true for all $t \in \mathbb{R} \geq 0$, it follows that

$$
\left(1-l_{\text {cor }}\right)=\left(1-l_{\text {meas }}\right) \cdot \operatorname{cor}_{\text {dyn }},
$$

whereby cor $_{\text {dyn }}$ is the dynamic correction function. That this is the case is exemplary shown with the leading edge. Figure 11 shows the general outline of the derivation. The Laplace transformed of Eqn. 6

$$
\chi_{\text {He,leading }}(s)=\frac{A_{\text {meas }} \cdot \ln \left(1-l_{\text {meas }}\right)}{s\left(\ln \left(1-l_{\text {meas }}\right)-s T\right)}
$$

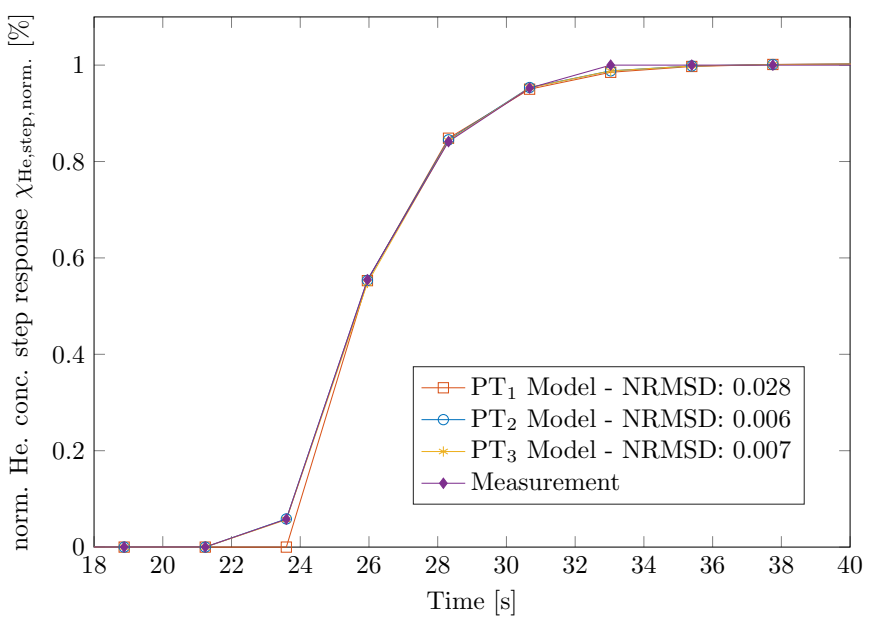

Figure 10: The figure shows an exemplary step response of the measurement setup compared to model responses of a $P T_{1}, P T_{2}$ and $P T_{3}$ transmission element response. The normalized root-mean-square deviation (NRMSD) of the models are given.

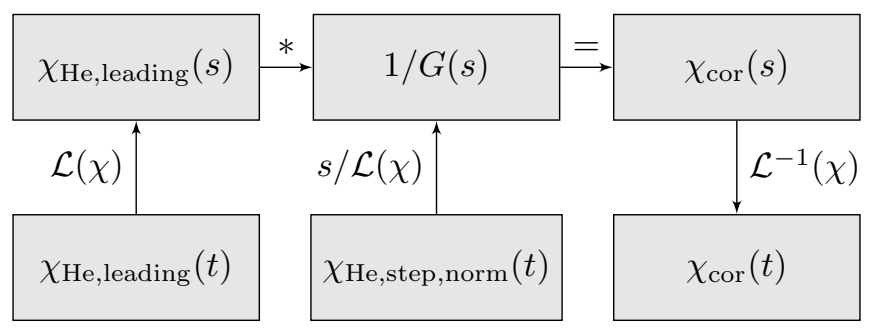

Figure 11: The figure shows the schematic of the determination of cor $r_{\text {dyn }}$. Whereby $\mathcal{L}$ is the Laplace transformation.

can be divided by Eqn. 9 to yield

$$
\begin{aligned}
\chi_{\mathrm{cor}}(s)= & \frac{A_{\text {meas }} \cdot \ln \left(1-l_{\text {meas }}\right)}{s\left(\ln \left(1-l_{\text {meas }}\right)-s T\right)} \\
& \cdot\left(s \cdot T_{1}+1\right)\left(s \cdot T_{2}+1\right) .
\end{aligned}
$$

Transferred back to the time domain using the inverse Laplace transformation $\mathcal{L}^{-1}\left(\chi_{\text {cor }}(s)\right)=\chi_{\text {cor }}(t)$ yields

$$
\begin{aligned}
\chi_{\mathrm{cor}}(t)= & A_{\text {meas }}\left(1-\left(1-l_{\text {meas }}\right)^{t / T}\right. \\
& \cdot\left(\frac{T_{1} \cdot \ln \left(1-l_{\text {meas }}\right)}{T}+1\right) \\
& \left.\cdot\left(\frac{T_{2} \cdot \ln \left(1-l_{\text {meas }}\right)}{T}+1\right)\right) .
\end{aligned}
$$

Introducing a function $f\left(T_{1}, T_{2}, l_{\text {meas }}, T\right)$ defined as

$$
f=\left(\frac{T_{1} \cdot \ln \left(1-l_{\text {meas }}\right)}{T}+1\right) \cdot\left(\frac{T_{2} \cdot \ln \left(1-l_{\text {meas }}\right)}{T}+1\right),
$$

Eqn. 14 can be expressed as

$$
\chi_{\mathrm{cor}}(t)=A_{\text {meas }}\left(1-\left(1-l_{\text {meas }}\right)^{t / T} \cdot f\right) .
$$

By equating the coefficients of Eqs.10 and 16, it follows that 


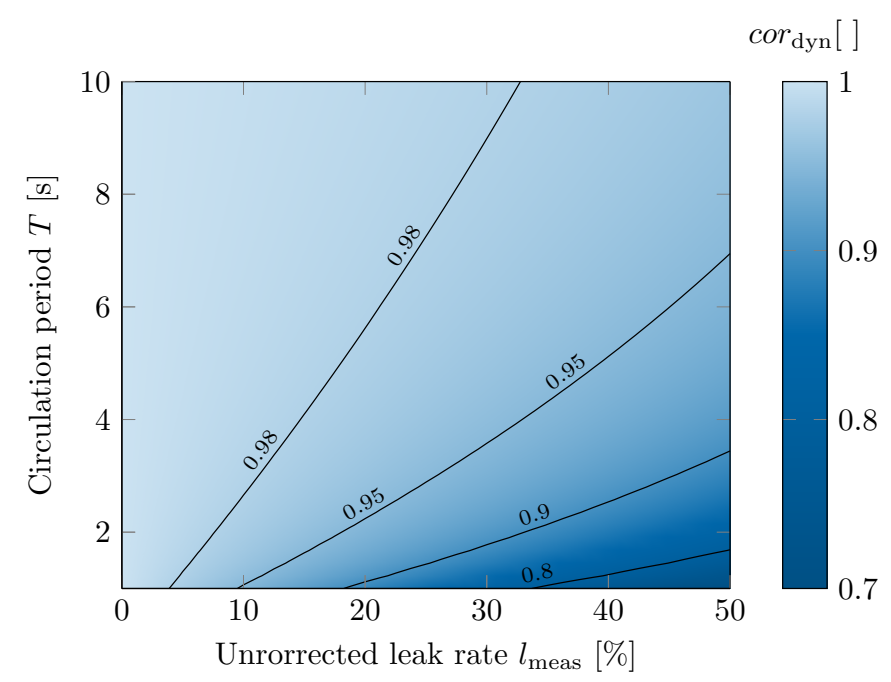

Figure 12: The figure shows the correction function cor $_{\text {dyn }}$ for different circulation period $T$ and leak rate $l_{\text {meas }}$ for a fixed $T 1=0.12 \mathrm{~s}$, $T 2=0.39 s$

$$
\begin{aligned}
\operatorname{cor}_{\mathrm{dyn}}= & f \\
= & \left(\frac{T_{1} \cdot \ln \left(1-l_{\text {meas }}\right)}{T}+1\right) \\
& \cdot\left(\frac{T_{2} \cdot \ln \left(1-l_{\text {meas }}\right)}{T}+1\right) .
\end{aligned}
$$

Having analytically found $c o r_{\mathrm{dyn}}$, it can be seen that $c o r_{\mathrm{dyn}}$ is measurable, since $T_{1}, T_{2}$ can be determined by fitting the step response shown in Fig. 10. The measurement of the circulation period $T$ is shown in sec. 3.3.1 the uncorrected leak rate $l_{\text {meas }}$ is determined from Eqs. 67. It must however be noted, that $T 1, T 2$ are determined empirically and must be determined for different maximum helium concentrations, so that the correction can be applied to all measurement scenarios. Fig. 12 shows the dependence of the correction function on the circulation period $T$ and leak rate $l_{\text {meas }}$ for a fixed $T 1, T 2$. It can be seen that the correction is most important for large leaks and short circulation times.

\subsubsection{Peripheral Leakage Measurement}

A further measurement that must be conducted, is the determination of the peripheral leak in the air system of a full circulation. By closing the receiver with a lid, this peripheral leak rate can be measured with the dynamic method. For the determination of this leakage, the trailing side of the concentration curve (Eqn. 7) is used. Therefore we can inject a small amount of helium and the formula shows, that the concentration should stay the same if $l=0$ because

$$
\lim _{l \rightarrow 0}\left(A \cdot \mathrm{e}^{\ln (1-l) \cdot \frac{t}{T}}\right)=A \cdot \mathrm{e}^{\ln (1) \cdot \frac{t}{T}}=A .
$$

For a complete circulation the peripheral leak rate has been determined to be cor $_{\text {peri }}=(2.4 \pm 0.3) \%$ per circulation. It is mainly caused by the blower. This leak rate correction value is used for the dynamic measurement. In contrast, the static measurement needs no leak correction, because there is no leak between receiver inlet and outlet.

\subsection{Signal Processing}

The mass spectrometer concentration data contains fluctuations, due to small concentration fluctuations in the test chamber and detector noise. Filtering of the data is not conducted, in order to preserve the uncertainty information of the data.

The background concentration of helium in the ambient air is subtracted from the data (Eqn. 50. This concentration can be higher in the laboratory than in the ambient air $(\approx 5.2 \mathrm{ppm})$, since helium is blown out into the lab. Without reducing this background, after 30 minutes of measurement the background helium concentration increased to $0.02 \%$. This corresponds to about $30 \%$ of the smallest measurement helium concentration. This background changes over time, making the data evaluation more complex. Therefore the helium background concentration was kept at a minimum of $<20$ ppm by opening large doors and a skylight in the laboratory.

\section{Uncertainty Analysis}

Uncertainty analysis is very important to assess the significance of the results. Therefore a sensitivity analysis to screen for relevant variables is conducted and the uncertainties are estimated according to the "Guide to the Expression of Uncertainty in Measurement" [4] using [8]. Finally the static and dynamic methods are validated with one another. All presented uncertainties are displayed with a $95 \%$ coverage factor.

\subsection{Sensitivity Analysis}

A sensitivity analysis has been conducted to get a better understanding of the most influential factors on the leak rate and on its type A measurement error. In order to examine the influence of a maximum of variables with a reasonable effort, an experiment using a D-optimized experiment plan has been conducted [17]. The amount of injected helium was hereby examined with more detail, since a great discrepancy between concentration values could have been an indication for a diffusion related uncertainty. Figure 14 shows both methods of measurement for two different helium concentrations. Since the results however lie well within the uncertainty of the measurement, the helium concentration is not a significant factor. A dependency of the uncertainty on the injected helium amount for the static method can be seen. This is expected, since an increase in helium concentration increases the signal to noise ratio of the measured signal. For an increase from $25 \mathrm{std} \mathrm{l} / \mathrm{min}$ to $50 \mathrm{stdl} / \mathrm{min}$ helium injection, the measurement uncertainty is reduced from $4.1 \%$ to $3.1 \%$ for the static method for the case of 8 of 13 attached air return tube (sec. 4.3). A difference in uncertainty due to the 


\begin{tabular}{|c|c|c|c|}
\cline { 2 - 3 } \multicolumn{1}{c|}{} & \multicolumn{2}{c|}{ Method } & Uncertainty \\
\cline { 2 - 3 } \multicolumn{1}{c|}{} & Static & Dynamic & Aype \\
\hline$l_{\text {meas }}$ & $(37.8 \pm 3.1) \%$ & $(38.3 \pm 2.1) \%$ & $\mathrm{~B} / \mathrm{B}$ \\
\hline cor $_{\text {peri }}$ & - & $(2.4 \pm 0.3) \%$ & $\mathrm{~A}$ \\
\hline$T$ & - & $(3.8 \pm 0.1) s$ & $\mathrm{~A}$ \\
\hline cor $_{\text {dyn }}$ & - & $(0.953 \pm 0.004)$ & $\mathrm{A}$ \\
\hline$l_{\text {cor }}$ & - & $(38.8 \pm 2.4) \%$ & $\mathrm{~A} / \mathrm{B}$ \\
\hline
\end{tabular}

Table 3: The table shows exemplary results of the variables necessary to determine the leak rate estimation for a helium injection of $50 \mathrm{std}$ $1 /$ min for the dynamic method with a $95 \%$ coverage factor and 8 of 13 attached air return tubes (see sec. 4.3 .

injected helium amounts of $25 \mathrm{std} 1 / \min$ to $50 \mathrm{std} 1 / \min$ could not be identified for the dynamic method. This can be explained by the fact, that the dynamic method is more robust against fluctuations in concentration levels, due to the fitting of the signal over time.

The ambient temperature, ambient air pressure and small variations in the air mass flow had no significant influence on the results and were not further examined.

\subsection{Uncertainty Estimation}

We will start with an uncertainty analysis of the static method, followed by the dynamic method, covering the necessary variables in the order as they are used.

Due to the measurement with the mass spectrometer a type B measurement uncertainty in helium concentration and time has to be considered. It is reduced by calibrating the mass spectrometer before each measurement campaign.

To calculate the type A measurement uncertainty of the static tracer gas method of $l_{\text {meas }}$, the standard deviation of the concentration measurements at both points of measurement are calculated and the uncertainty is propagated using Gaussian error propagation. Since the static measurement is independent in time, only the type B measurement error in helium concentration must be considered.

The variables necessary to determine the leak rate $l$ are shown in Table 3 with their measurement uncertainties for one specific measurement. To calculate the type A measurement uncertainty of $l_{\text {meas }}$ of the dynamic measurement, first the type A uncertainty is determined from the goodness of fit of both the leading as well as the trailing edge. Since this leak rate determination is independent of the maximum helium concentration but dependent on time, the only type B measurement uncertainty which has to be considered is the timing uncertainty. Since the time in Eqs. 677 is divided by the circulation period a linear clock drift would be canceled out. Since the fitting is robust against small fluctuations in time, the type B measurement uncertainty of the mass spectrometer is not introduced.

The uncertainty in the circulation period $T$ has only a very small type A measurement error, due to large statis- tics made possible by the automated measurement setup and fitting as well as the short circulation period.

A further uncertainty occurs due the determination of the transfer function $c_{\mathrm{dyn}}$. It arises due to the difficult nature of the experiment. By not introducing a perfect step function into the system when determining the transfer function, the transfer function will most likely be overestimated. The type A uncertainty is determined using the standard deviation of the ten smallest correction values.

The uncertainty of $l_{\text {cor }}$ is calculated by error propagation.

\subsection{Validation of Measurement Methods}

In order to validate the static and dynamic measurement method, the leak rate for four different measurements scenarios was measured using the dynamic and the static measurement method. The scenarios with a fixed unknown leak rate were created by closing the receiver with a lid and removing a certain amount of the 13 return tubes. Each static measurement which is shown consists of 8 minutes of helium injection, after two minutes the measurement starts. Every 60 seconds the measurement point is switched, in total 5 minutes of the measurement data is used as indicated in Fig. 13. For each dynamic measurement point five peaks of 60 seconds each is followed by a pause, resulting in 5 minutes of active measurement, making it comparable to the static measurement. Figure 6 shows three of such 60 second peaks.

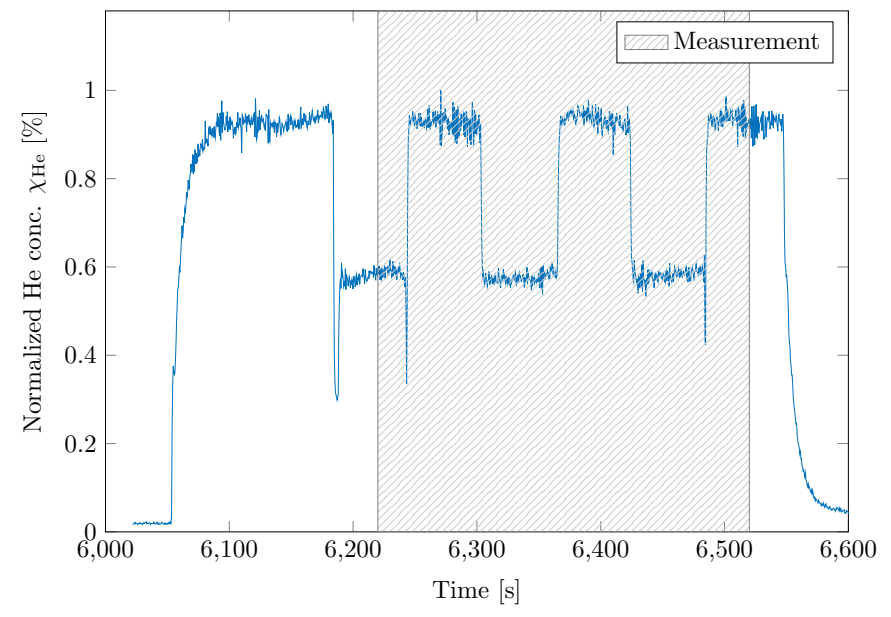

Figure 13: The figure shows the normalized raw data for a helium injection of $50 \mathrm{std} 1 / \mathrm{min}$ for the static method with a $95 \%$ coverage factor and 8 of 13 attached air return tubes.

The results are shown in Fig. 14 . Since both measurements of the leak rate are taken under the same experimental conditions, they should yield the same result. This is in close agreement with the measurements, since they lie well within the uncertainty bounds. Because the two measurement methods have roughly the same measurement error, a correction function was not introduced and the measurement methods can be considered validated. 


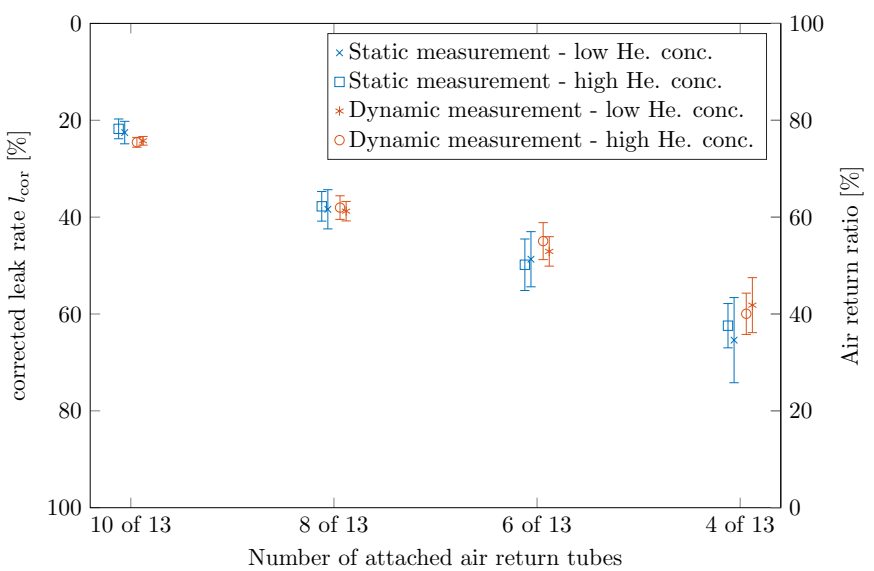

Figure 14: The leak rate per circulation was measured using the static as well as the dynamic tracer gas measurement with a closed receiver configuration (see Fig. 5) for four different scenarios with a certain number of return tubes attached. The measurement was conducted for two different helium injection quantities (25/50 std $1 / \mathrm{min}$ ) and are presented with a $95 \%$ coverage factor.

\section{Exemplary Results of the Model}

An exemplary measurement result at the model was conducted at $\dot{m}_{i n}=(0.247 \pm 0.008) \mathrm{kg} / \mathrm{s}$. The mass flow measurement was conducted without helium present, since helium interferes with the mass flow measurement. The same measurement conditions as in sec. 4.3 were applied. The dynamic fit result, using Eqs. 6 and 7, is shown for

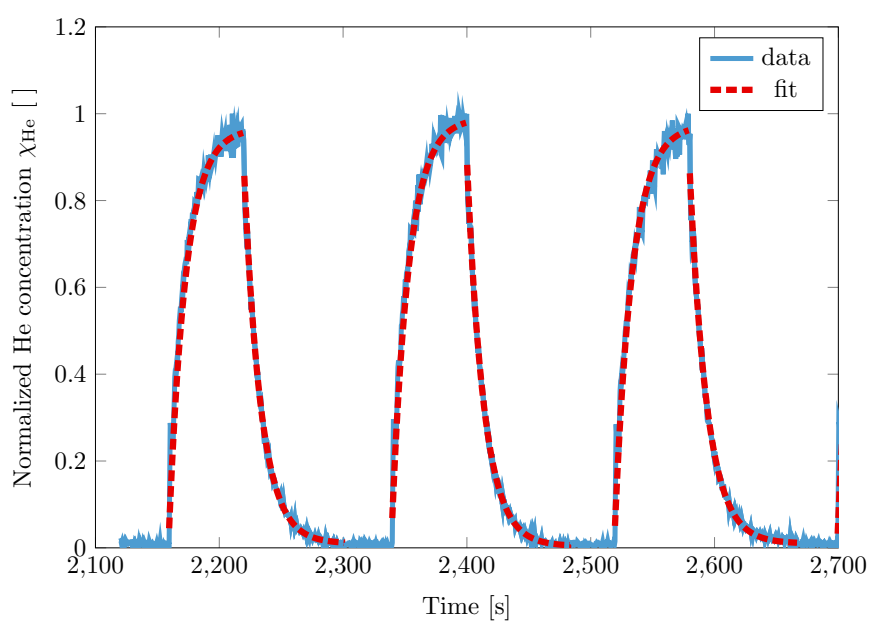

Figure 15: The leak rate per circulation was measured using the dynamic tracer gas measurement. The concentration signal was fitted with Eqs. 6] and 7

three measurements in Fig. 15 .

The leak rate was determined to be $l_{\text {stat }}=(36.1 \pm 2.3) \%$ and $l_{\mathrm{dyn}}=(34.5 \pm 3.6) \%$. This corresponds to an air return ration of $A R R_{\text {stat }}=(63.9 \pm 2.3) \%$ and $A R R_{\text {dyn }}=$ $(65.5 \pm 3.6) \%$. The uncertainties are given with a $95 \%$ coverage factor. Both methods deliver values which lie well within their uncertainty bounds.

\section{Conclusion}

An environmentally friendly, quantitative leak rate measurement technique suitable for large scale and hot air flows was developed. A tracer gas method using helium was chosen. The circular nature of the measurement environment allowed us the development of a dynamic tracer gas measurement technique. This additionally technique allows us the validation of the leak measurement and has the further benefit of needing only one measuring point.

The two methods were successfully validated for four fixed leak rates. The measurement results of the two measuring methods lie within the error of measurement. The absolute uncertainty is smaller for small leak rates $\left(l_{\text {stat }}=\right.$ $\left.(21.8 \pm 2.0) \%, l_{\mathrm{dyn}}=(24.5 \pm 1.0) \%\right)$ than for larger ones $\left(l_{\text {stat }}=(62.4 \pm 4.6) \%, l_{\text {dyn }}=(60.0 \pm 4.3) \%\right)$. This is to be expected, due to a better signal to noise relationship at lower leak rates and the fact that the uncertainties of the corrections are relative to the leak rate.

One exemplary measurement has been presented, with a mass rate of $\dot{m}=(0.247 \pm 0.008) \mathrm{kg} / \mathrm{s}$ resulting in a leak rate of $l_{\text {stat }}=(36.1 \pm 2.3) \%$ and $l_{\mathrm{dyn}}=(34.5 \pm 3.6) \%$.

Due to the successful measurement at laboratory scale it can be expected, that the leak rate measurements or alternatively the air return ratio measurements at the Solar Tower Jülich will yield reliable results.

\section{Acknowledgments}

This work was carried out with financial support from the Ministry of Innovation, Science and Research of the State of North Rhine-Westphalia (MIWF NRW), Germany under contract 323-2010-006 (Start-SF).

\section{AppendixA. Bibliography}

[1] Ahlbrink, N., Andersson, J., Diehl, M., Pitz-Paal, R., 2013. Optimization of the mass flow rate distribution of an open volumetric air receiver. Journal of Solar Energy Engineering 135 (4), 041003.

URL http://dynamicsystems.asmedigitalcollection.asme. org/article.aspx?articleid=1700705

[2] Cui, S., Cohen, M., Stabat, P., Marchio, D., 2015. Co 2 tracer gas concentration decay method for measuring air change rate. Building and Environment 84, 162-169.

URL http://www.sciencedirect.com/science/article/pii/ S0360132314003606

[3] Cussler, E. L., 2009. Diffusion: mass transfer in fluid systems. Cambridge university press.

[4] des Poids et Mesures, B. I., électrotechnique internationale, C., internationale de normalisation, O., 1995. Guide to the Expression of Uncertainty in Measurement. International Organization for Standardization.

URL http://www.iso.org/iso/home/store/catalogue_ics/ catalogue_detail_ics.htm?csnumber $=50461$

[5] Forster, P., Ramaswamy, V., Artaxo, P., Berntsen, T., Betts, R., Fahey, D. W., Haywood, J., Lean, J., Lowe, D. C., Myhre, G., et al., 2007. Changes in atmospheric constituents and in radiative forcing. chapter 2. In: Climate Change 2007. The Physical Science Basis. Teruyuki Nakajima, Veerabhadran Ramanathan. URL http://www.ipcc.ch/pdf/assessment-report/ar4/wg1/ ar4-wg1-chapter2.pdf 
[6] Ghazi, C., Marshall, J., 2014. A co 2 tracer-gas method for local air leakage detection and characterization. Flow Measurement and Instrumentation 38, 72-81.

URL http://wwW.sciencedirect.com/science/article/pii/ S0955598614000612

[7] ISO12569, 2012. Thermal performance of buildings and materials - determination of specific airflow rate in buildings - tracer gas dilution method. International Organization for Standardization.

URL https://www.iso.org/obp/ui/\#iso:std: iso: 12569

[8] Kirkup, L., Frenkel, R. B., 2006. An Introduction to Uncertainty in Measurement: Using the GUM (Guide to the Expression of Uncertainty in Measurement). Cambridge University Press.

[9] Koch, D., 2003. Sf6 properties, and use in mv and hv switchgear. Cahier technique (188).

[10] Koll, G., Schwarzboezl, P., Hennecke, K., Hartz, T., Schmitz, M., Hoffschmidt, B., 2009. The solar tower juelich-a research and demonstration plant for central receiver systems. Proceedings SolarPACES.

URL http://elib.dlr.de/60306/

[11] Robinson, P., Latzin, P., Verbanck, S., Hall, G. L., Horsley, A., Gappa, M., Thamrin, C., Arets, H. G., Aurora, P., Fuchs, S., et al., 2013. Consensus statement for inert gas washout measurement using multiple and single breath tests. European Respiratory Journal, erj00697-2012.

URL http://www.ncbi.nlm.nih.gov/pubmed/23397305

[12] Romero, M., Steinfeld, A., 2012. Concentrating solar thermal power and thermochemical fuels. Energy \& Environmental Science 5 (11), 9234-9245. URL http://dx.doi.org/10.1039/C2EE21275G

[13] Tang, J., Noakes, C., Nielsen, P., Eames, I., Nicolle, A., Li, Y., Settles, G., 2011. Observing and quantifying airflows in the infection control of aerosol-and airborne-transmitted diseases: an overview of approaches. Journal of Hospital Infection 77 (3), 213-222.

URL http://www .ncbi.nlm.nih.gov/pubmed/21194796

[14] Tellez, F., Romero, M., Heller, P., Valverde, A., Reche, J., Ulmer, S., Dibowski, G., 2004. Thermal performance of "solair $3000 \mathrm{kWth}$ " ceramic volumetric solar receiver. 12th International Symposium Solar Power and Chemical Energy Systems. URL http://www.sciencedirect.com/science/article/pii/ S0038092X11000302

[15] Vogel, W., Kalb, H., 2010. Large-scale solar thermal power: technologies, costs and development. John Wiley \& Sons.

[16] von Storch, H., Stadler, H., Roeb, M., Hoffschmidt, B., 2015. Efficiency potential of indirectly heated solar reforming with open volumetric solar receiver. Applied Thermal Engineering. URL http://dx.doi.org/10.1016/j.applthermaleng.2015. 05.026

[17] Wember, T., 2008. Technische statistik und statistische versuchsplanung.

\section{Glossary}

Ar Argon

ARR Air return ratio

$\boldsymbol{A} \boldsymbol{R} \boldsymbol{R}_{\text {dyn }}$ Air return ratio measurement with dynamic method

$\boldsymbol{A R R}_{\text {stat }}$ Air return ratio measurement with static method

$\mathrm{CO}_{2}$ Carbon dioxide

cor $_{\text {peri }}$ Peripheral leak correction

$\boldsymbol{c o r}_{\text {dyn }}$ Dynamic error correction

G Transfer function

$\mathbf{H}^{*}$ Forming gas

He Helium

$l$ Leak rate

$\boldsymbol{l}_{\mathbf{d y n}}$ Leak rate measured with the dynamic method

$\boldsymbol{l}_{\text {stat }}$ Leak rate measured with the static method

$\boldsymbol{L}$ Laplace transform

$\dot{m}$ air mass flow

Ne Neon

NRMSD Normalized root-mean-square deviation

PIV Particle image velocimetry

$\mathbf{P T}_{\mathbf{n}} \mathrm{n}^{\text {th }}$-order lag element

s Complex number frequency

SF $_{6}$ Sulfur hexafluoride

T Circulation period

$\mathbf{T}_{\mathbf{1 , 2}}$ Time constants of the PT2 element

$\chi_{\mathbf{H e}}$ Helium concentration

$\chi_{\text {step }}$ Concentration step response 